Recepción: 20 / 12 / 2017

Aceptación: 15 / 02 / 2018

Publicación: 21 / 04 / 2018

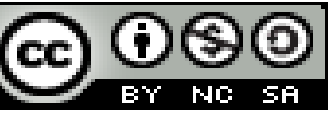

Ciencias Administrativas

Artículo de Revisión

\title{
Sistemas de franquicias como parte de los procesos de globalización económica y su contribución al desarrollo de la provincia de Manabí
}

\section{Franchising systems as part of the processes of economic globalization and its contribution to the development of the province of Manabi}

\section{Sistemas de franquia como parte dos processos de globalização econômica e sua contribuição para o desenvolvimento da província de Manabí}

\author{
Miguel A. Baque-Cantos I \\ miguel.baque@hotmail.com \\ José L. Merino-Murillo II \\ joselu_merino28@hotmail.com
}

\author{
Adela del Jesús Lucio Pillasagua III \\ adel_luci@yahoo.es \\ Antonio E. Osejos-Vásquez ${ }^{\text {IV }}$ \\ aeov2@yahoo.es
}

Correspondencia: miguel.baque@unesum.edu.ec

I. Ingeniero Comercial: Universidad Técnica de Manabí; Diplomado en Autoevaluación y Acreditación Universitaria. Universidad Aconcagua República de Chile; Magister. Docencia Universitaria e Investigación Educativa. Universidad Nacional de Loja; Docente Principal Titular Universidad Estatal del Sur de Manabí, Jipijapa, Ecuador.

II. Ingeniero Comercial: Universidad Laica Eloy Alfaro de Manabí; Magister en Finanzas y Comercio Internacional: Universidad Laica Eloy Alfaro de Manabí; Docente: Contratado: Tiempo completo de la Carrera Comercio Exterior Universidad Estatal del Sur de Manabí, Jipijapa, Ecuador.

III. Ingeniera en Auditoria Universidad Estatal del Sur de Manabí- Facultad de Ciencias Económicas; Magister en Gerencia Educativa Universidad Estatal del Sur de Manabí; Docente: Contratado: Tiempo completo de la Carrera Comercio Exterior y Auditoria Universidad Estatal del Sur de Manabí, Jipijapa, Ecuador.

IV. Ingeniero en Alimentos: Universidad Laica Eloy Alfaro de Manabí; Magister en Finanzas y Comercio Internacional Universidad Laica Eloy Alfaro de Manabí y Docente: Contratado: Tiempo completo de la Carrera Comercio Exterior Universidad Estatal del Sur de Manabí, Jipijapa, Ecuador. 


\section{Resumen}

Las franquicias constituyen un modelo muy rentable de negocios, porque generan empleo, satisfacen las demandas de los consumidores de una manera rápida y confiable y en muchos casos a bajo costo, tienen la capacidad de expandirse rápidamente y pueden convertir un negocio prospero en un modelo exitoso de negocios a gran escala, casos famosos en el mundo están a la vista Mc Donald's, Pizza Hut, Burger King, y en Ecuador Disensa, Farmacias Cruz Azul, entre otras. La investigación es de tipo documental no experimental. Entre las conclusiones más relevantes es que no existe una ley de franquicias en el

Ecuador, no existe información de la contribución del desarrollo económico de la provincia de Manabí y las asociaciones que agrupan a las franquicias no tienen estadísticas actualizadas sobre la cantidad de estos establecimientos a nivel provincial. Como recomendaciones es necesaria la redacción de una ley de franquicias que norme y regule esta actividad, y que las asociaciones que agrupan esta actividad manejen información más detallada a nivel provincial.

Palabras claves: Franquicia, empleo, desarrollo, consumidores, negocios. 


\begin{abstract}
The franchises are a very profitable business model, because they generate employment, satisfy the demands of consumers in a fast and reliable and in many cases at low cost, have the ability to expand quickly and can turn a successful business into a successful model large scale business, famous cases in the world are in sight Mc Donald's, Pizza Hut, Burger King, and in Ecuador Disensa, Cruz Azul Pharmacies, among others. The research is non-experimental documentary type. Among the most relevant conclusions is that there is no franchise law in the Ecuador, there is no information on the contribution of the economic development of the province of Manabí and the associations that group the franchises do not have updated statistics on the number of these establishments at the provincial level. As recommendations, it is necessary to draft a franchise law that regulates and regulates this activity, and that the associations that group this activity handle more detailed information at the provincial level.
\end{abstract}

Keywords: Franchising, employment, development, consumers, business. 


\section{Introducción.}

La Franquicia, es un modelo de negocios exitoso universalmente conocido por su creíble y académico concepto, digno de apoyar su difusión, y porque dentro de su contexto involucra las fases claves de todo plan de negocios, como estudio de mercado, estudio técnico, estudio financiero y estudio de la rentabilidad, y la ventaja de que siendo su concepto ya probado implica menor riesgo para la inversión (Martinez Tubay \& Alvarez Rivas, 2015). Toda iniciación de un negocio independientemente de su tamaño, requiere la evaluación de su entorno, unos más que otros realizan grandes proyectos de inversión para determinar si es factible o no llevar a cabo dicho negocio, hay otros que con pocos insumos y mucha intuición en los negocios logran también establecerlos. En este caso las franquicias llegaron en un momento clave para cambiar el mercado, en donde la satisfacción al cliente en el menor tiempo posible fue, es y sigue siendo su objetivo principal.

El mercado ecuatoriano se está abriendo a un modelo de crecimiento empresarial, junto al creciente número de establecimientos extranjeros localizados en Ecuador, se está sumando una expansión de la franquicia local en las principales ciudades del país. Este proceso viene apoyado por la apertura de malls o grandes centros comerciales, alrededor del país que ofrecen grandes posibilidades para el desarrollo del modelo, y que reúnen los requisitos que los franquiciadores reclaman para sus negocios, alto número de visitantes, facilidad para la apertura de locales y una amplitud de horario comercial (Vizcaino Agama, 2009).

La franquicia como es un método probado genera un caso de Éxito del 90\% ya que se aporta al franquiciado el conocimiento y recursos necesarios para mantener una estabilidad empresarial, además de que las franquicias son una herramienta generadora de empleos formales, en donde se calcula que por cada franquicia creada se genera entre 8 y 10 puestos de trabajo en promedio y es en 
Sistemas de franquicias como parte de los procesos de globalización económica y su contribución al desarrollo de la provincia de Manabí

esta área en donde se crea un desarrollo integral en un país. La creación de fuentes de empleo dignas es el paso fundamental si queremos que nuestras comunidades del interior de la república se desarrollen y salgan adelante (Lopez, 2016, p. 7-8) (VÁSQUEZ GÓMEZ, 2017).

En Ecuador actualmente operan aproximadamente 152 franquicias nacionales e internacionales, en 1.200 establecimientos que dan empleo a aproximadamente a 10.000 personas, y que alcanzan una facturación de casi 900 millones de dólares (600 millones de euros) (Organización Mundial de Comercio, 2012) (Guerrero, 2015).

Lo evidente es el éxito que han tenido las franquicias como modelo de negocio para el emprendimiento. Es decir, todas las cadenas del sector de comida con su sistema han logrado adjudicarse un espacio en el mercado e incrementar la economía en el país, por ejemplo una familia ecuatoriana destina en promedio el 5,3\% de su ingreso para el consumo en este rubro, lo que equivale sumando todo el consumo nacional a un poco más de 40 millones de dólares al mes (¡500 millones al año!) (Martinez Tubay \& Alvarez Rivas, 2015). Las franquicias nacieron en el negocio de la comida, los consumidores buscaban alternativas para consumir alimentos en el menor tiempo posible, y por ello se establecieron estos estilos de negocios para darle al consumidor rapidez, eficiencia a bajo costo y en el menor tiempo posible, casos mundialmente famosos son la prueba de ello (Mc Donald's, Pizza Hut, entre otros).

Existen beneficios y riesgos calculados tanto para los empresarios, los consumidores y el Estado:

- Desde el punto de vista del empresario privado, obtener una franquicia le resulta atractivo debido al bajo riesgo que esto representa al estar respaldado por una marca global, exitosa y prestigiosa. 
Miguel A. Baque-Cantos; José L. Merino-Murillo; Adela del Jesús Lucio Pillasagua; Antonio E. Osejos-Vásquez

- Para los consumidores es ventajoso conocer el valor agregado de la oferta de estos negocios y tener variedad de opciones para satisfacer sus gustos y preferencias.

- Para el Estado, el ingreso de estas inversiones beneficia sin duda a la balanza de pagos, generando así un crecimiento importante en el PIB. Por tal motivo, muchos países lo consideran una vía importante de recursos y de generación de empleo, a la vez que se incrementa la contribución fiscal (Ledesma Cárdenas \& Alcívar Rodríguez, 2015).

El desarrollo económico de un pueblo debe basarse en el proceso de transformación de la economía y de la sociedad, que está encaminada a superar los problemas y desafíos existentes, para poder optimizar las situaciones de vida de su población mediante un trabajo decidido y concertado entre los diferentes agentes socioeconómicos locales, públicos y privados, para el beneficio más eficiente y sostenible de los recursos, mediante el fomento de las capacidades de emprendimiento industrial y la creación de un ambiente innovador en la parroquia (Pinargote Mendoza, 2017).

La provincia de Manabí a pesar de tener grandes recursos turísticos sostenibles presenta dificultades actualmente con la calidad del servicio que oferta, careciendo de propuestas que motiven a los visitantes, cuestión que incide negativamente sobre la economía, según menciona Valencia (2011) el sector turístico de la provincia presenta bajos niveles de visitantes, debido a la calidad del servicio que se brinda en la provincia, lo que trae consigo un aumento de costos en este sector y una disminución de las ganancias que afecta directamente la economía de la provincia y del país (Bayas Escudero, Chancay Pozo, \& Delgado Mejía, 2015).

Las playas manabitas han sido relegadas a un segundo plano debido a la tendencia histórica del turismo nacional por visitar Esmeraldas y Salinas, pero gracias a diversos factores vinculados a la actividad turística, las playas de Manabí han llegado a constituir las nuevas opciones de turismo. 
Sistemas de franquicias como parte de los procesos de globalización económica y su contribución al desarrollo de la provincia de Manabí

Entre estos factores podemos mencionar: la construcción y mantenimiento de la Base Aérea temporal de los norteamericanos en la ciudad de Manta, la construcción del edificio de la Asamblea Nacional Constituyente en la ciudad de Montecristi, la ampliación y concesión del puerto de Manta, la construcción de la vía Manta - Manaos, la construcción de la Refinería de petróleo en el Aromo, entre los más importantes (Tapia Arcos, 2009).

\section{Materiales y métodos.}

La investigación se enmarca dentro del tipo documental no experimental. Documental, ya que fueron utilizado medios electrónicos, artículos de revistas, páginas web, entre otros elementos, para alimentar la investigación del objeto de estudio. No experimental, ya que no se está realizando ningún tipo de estudio de campo, se tomaron las ideas expresadas por los autores citados en el presente trabajo sin alterar sus planteamientos, solo se han hecho análisis de las opiniones aquí expresadas y los autores citados, han sido utilizados como referencias bibliográficas. 


\section{Resultados.}

\section{Imagen $\mathbf{N}^{\circ}$ 1: Origen de marcas}

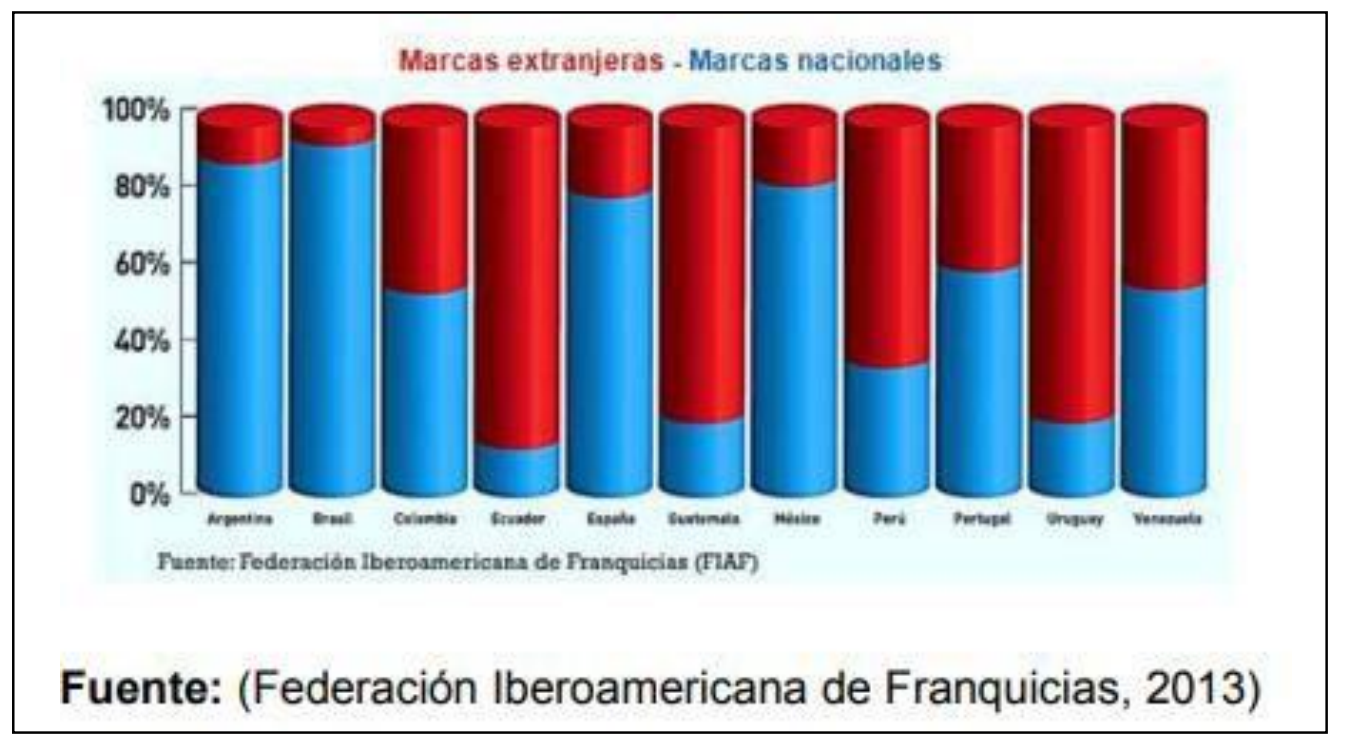

Fuente: (Rubio, 2014).

En la imagen $\mathrm{N}^{\circ}$ 1, se observa que para el año 2013, menos del $20 \%$ de las franquicias existentes en Ecuador, el origen de la franquicia es nacional, más bien son de marcas o ideas originarias de países extranjeros. Ecuador está en el último puesto de la tabla superado ampliamente por Argentina, Brasil, Colombia, México, Venezuela, entre otros. 
Sistemas de franquicias como parte de los procesos de globalización económica y su contribución al desarrollo de la provincia de Manabí

Imagen $\mathbf{N}^{\circ}$ 2: Numero de franquicias por países

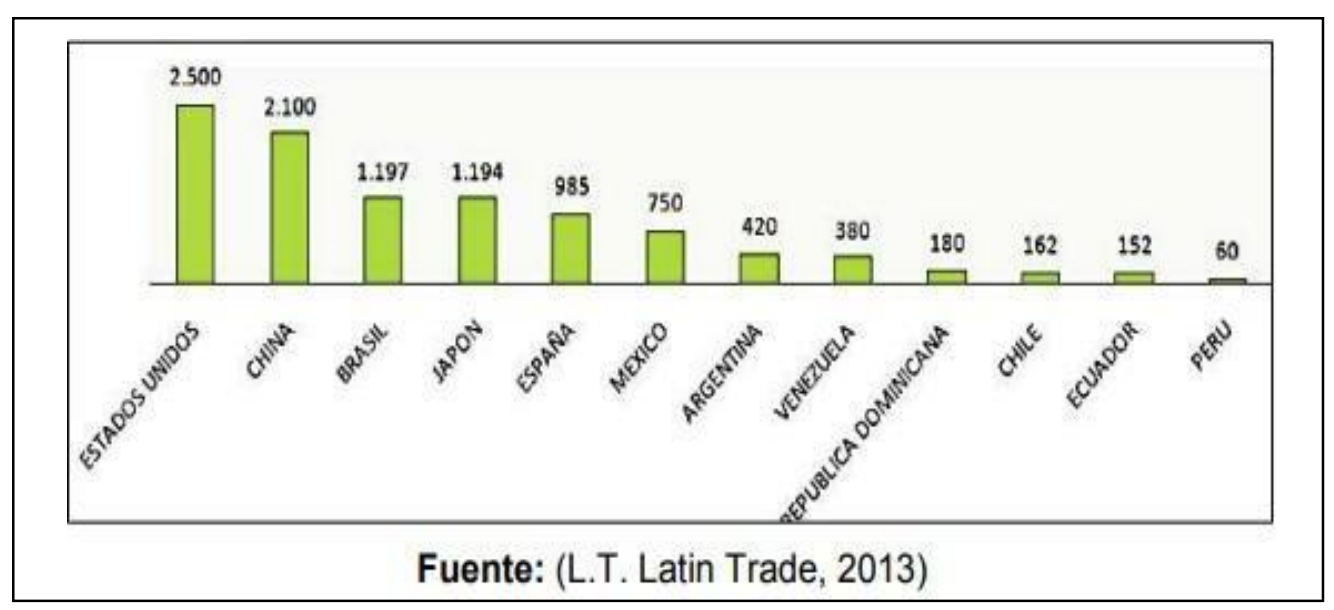

Fuente: (Rubio, 2014).

En la imagen $\mathrm{N}^{\circ} 2$, se sigue observando una tendencia donde Ecuador es superado esta vez en el número de franquicias que existen en el país, con un total para la fecha de 152, solo por debajo de Perú que para el momento del estudio tenía 60 franquicias. 
Miguel A. Baque-Cantos; José L. Merino-Murillo; Adela del Jesús Lucio Pillasagua; Antonio E. Osejos-Vásquez

Imagen $\mathbf{N}^{\circ}$ 3: Tipos de franquicias en Ecuador

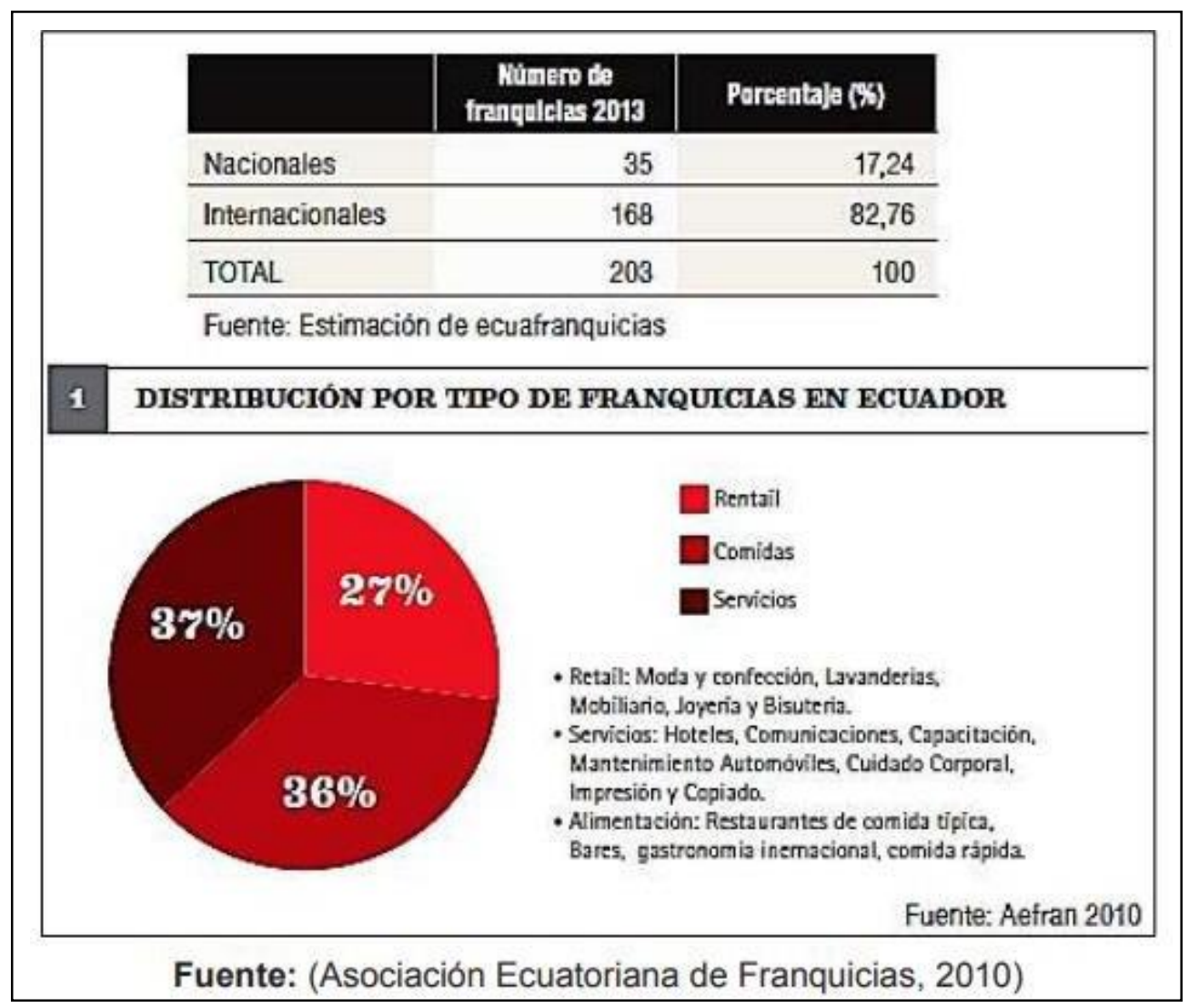

Fuente: (Rubio, 2014).

En la imagen $\mathrm{N}^{\circ} 3$ se observa la distribución por tipo de franquicias que existen en Ecuador, el 36\% de las franquicias son del área de Fast Food (comida rápida), el 37\% representan servicios varios y el $27 \%$ servicios de moda, lavandería, entre otros. 
Sistemas de franquicias como parte de los procesos de globalización económica y su contribución al desarrollo de la provincia de Manabí

Imagen $\mathbf{N}^{\circ}$ 4: franquicias en Iberoamérica

\begin{tabular}{|c|c|c|c|c|c|c|c|c|}
\hline PAIS & $\begin{array}{c}\mathrm{N}^{\circ} \\
\text { MARCAS }\end{array}$ & NACIONAL & $\%$ E & EXTRANJERA & $\%$ & $\begin{array}{l}\text { FACTURACIÓN } \\
\text { millones USD }\end{array}$ & $\begin{array}{l}\text { INIDADES } \\
\text { frang + } \\
\text { proplas }\end{array}$ & PERSONAL \\
\hline ARGENTINA & 400 & 360 & 90 & 40 & 10 & $2.781,03$ & 18.500 & 150.000 \\
\hline BRASIL & 1.197 & 1.065 & 89 & 132 & 11 & $26.983,00$ & 65.553 & 586.000 \\
\hline ECUADOR & 168 & 25 & 15 & 143 & 85 & 890,00 & 1.260 & 12.722 \\
\hline ESPANÃ & 804 & 570 & 81 & 152 & 19 & $34.871,77$ & 56.039 & 227.271 \\
\hline GUATEMALA & 200 & 35 & 18 & 165 & 82 & - & 4.500 & 45.000 \\
\hline MEXICO & 750 & 615 & 70 & 135 & 18 & $9.000,00$ & 60.000 & 610.000 \\
\hline PERU & 100 & 30 & 30 & 70 & 70 & 450,00 & 264 & 14.000 \\
\hline PORTUGAL & 481 & 245 & 51 & 236 & 49 & $5.574,00$ & 10.700 & 63.100 \\
\hline VENEZUELA & 352 & 225 & 64 & 127 & 36 & $5.810,00$ & 13.500 & 54.200 \\
\hline TOTALES & 4.082 & 2.810 & - & 1.160 & $\cdot$ & $83.369,80$ & 230.316 & 1.612 .293 \\
\hline \multicolumn{9}{|c|}{ Fuente: Federación Ibero Americana de Franquicias } \\
\hline
\end{tabular}

Fuente: (Silva, 2010).

Entre las contribuciones que representan las franquicias para el desarrollo de los países están:

- En la capacidad que tienen de rediseñar los sistemas de bienes y servicios, para mejorar sus estándares de calidad conociendo los gustos de los consumidores.

- Tienden a multiplicarse rápidamente por la geografía del país donde se establecen, generalmente en las principales ciudades, en donde realizan estudios de mercados para establecer la mejor posición de su ubicación, en muchos casos una franquicia reconocida puede tener más de 10 locales establecidos en una provincia.

- Son generadores de empleo tanto directos como indirectos, dentro de este mismo estudio se revela que en Ecuador se generan por este medio aproximadamente 10.000 puestos de trabajo, esto sin sumarle el total de puestos de trabajo indirectos por actividades secundarias. 
- El impacto economico y social generados por las franquicias determina un elevado grado de desarrollo sobre todo el ambito labral y comercial, debido principalmete a las ingentes cantidades de dinero que se mueven en el sistema (Silva, 2010).

- Contribuyen al desarrollo del país por su capacidad de pagar impuestos locales y nacionales, su actividad económica tributa tanto al producto interno bruto (PIB) como al producto territorial bruto del país (PTB).

\section{Conclusiones.}

No existe hasta los momentos una Lay de Franquicias en el Ecuador.

El Instituto Nacional de Estadísticas y Censos de Ecuador (INEC), no tiene información desagregada de la cantidad de empleos que generan las franquicias y los ingresos que se generan de este tipo de comercio.

En la provincia de Manabí no hay registros de la cantidad de franquicias tanto nacionales y extranjeras establecidas, mucho menos la cantidad de puestos de trabajo que generan.

La Asociación Ecuatoriana de Franquicias (AEFRAN), no posee estadísticas de la cantidad de franquicias establecidas hasta la fecha en Ecuador.

En los múltiples trabajos consultados en donde se habla de la provincia de Manabí, no existe información real de la cantidad de franquicias y otros aspectos económicos, solo se concentran en las características geográficas y económicas de la provincia y propuestas de proyectos factibles de establecer franquicias o convertir en franquicias a negocios ya establecidos. 
Sistemas de franquicias como parte de los procesos de globalización económica y su contribución al desarrollo de la provincia de

\section{Recomendaciones.}

Es necesaria la redacción de una ley de franquicias en el país que norme y regule su funcionamiento.

Todas las asociaciones que agrupen a franquicias deberían tener en sus portales información estadística de interés sobre este tipo de comercios.

La cámara de comercio de Manabí o el ente encargado de agrupar a los comerciantes de la provincia deberían tener también algún tipo de información estadística, sobre esta materia.

Buscar un aprovechamiento máximo de los sistemas de franquicias en el Ecuador, valiéndose principalmente de la dolarización como eje motriz de las mismas, ya que este sistema económico constituye un aliciente eficaz y probado para atraer inversión extranjera, en virtud de que esta moneda aún se constituye como el referente monetario a nivel mundial (Silva, 2010).

\section{Bibliografía.}

Bayas Escudero, J., Chancay Pozo, R., \& Delgado Mejía, M. (2015). Gestión operativa de restaurantes para el mejoramiento de los servicios gastronómicos en la parroquia Calceta de la provincia de Manabí. Calceta: ESPAM.

Guerrero, M. (2015). El empleo directo e indirecto que generan las franquicias nacionales en la ciudad de Quito.

Ledesma Cárdenas, M., \& Alcívar Rodríguez, B. (2015). La franquicia como alternativa para cubrir la demanda en el sector de la salud de Guayaquil. Guayaquil: UG.

Martinez Tubay, M., \& Alvarez Rivas, H. (2015). La Franquicia como plan de negocios Alternativa para el Emprendimiento en el Ecuador. Guayaquil: UG.

Pinargote Mendoza, R. (2017). Comercializacion de empanadas de platano y su impacto en el desarrollo socioeconomico de la parroquia Ayacucho del Canton Santa Ana. Jipijapa: UNESUM. 
Miguel A. Baque-Cantos; José L. Merino-Murillo; Adela del Jesús Lucio Pillasagua; Antonio E. Osejos-Vásquez

Rubio, E. (2014). Análisis del sistema de franquicias como factor de desarrollo económico en el Ecuador. Guayaquil: UG.

Silva, A. (2010). Efectos economicos y sociales de las franquicias en el Ecuador. Quito: UDLA.

Tapia Arcos, D. (2009). Proyecto de factibilidad para la creación de una Hostería en la parroquia Santa Marianita, cantón Manta, provincia de Manabí. Quito: ESPE.

VÁSQUEZ GÓMEZ, A. (2017). Análisis de las franquicias existentes en la ciudad de Esmeraldas. Esmeraldas: PUCE.

Vizcaino Agama, D. (2009). Proyecto de factibilidad para establecer una sucursal de la franquicia Pizza Hut en Manta. Quito: Universidad Internacional SEK. 